\title{
UMA PROPOSTA DE MODELO DE INTERFACE INTEROPERÁ VEL PARA WEB, TV DIGITAL E DISPOSITIVOS MÓVEIS
}

\author{
Maria Lúcia Kroeff Barbosa; PGIE, UFRGS; malukroeff@ yahoo.com.br \\ Valter Roesler; Instituto de Informática, UFRGS; roesler@inf.ufrgs.br \\ Eliseo Reategui; PGIE, UFRGS; eliseoreategui@gmail.com
}

Resumo. Este artigo apresenta um modelo de interface para cursos educacionais interoperáveis entre web, TV Digital e dispositivos móveis. Levando-se em consideração as dificuldades atuais para padronizar conteúdo entre as três plataformas e os requisitos na elaboração de interfaces satisfatórias para conteúdo educacional, o principal objetivo é desenvolver um modelo de interface para objetos de aprendizagem que mantenham, praticamente, as mesmas características visuais e interativas independente de onde forem acessados. Para validar a proposta, foi desenvolvido um curso sobre saúde e qualidade de vida e desenvolveu-se uma interface interoperável que visa contemplar essas questões.

Palavras-chave: interface, educação, interoperabilidade

\section{A proposal of an interoperable interface model for Web, Digital TV and mobile devices}

Abstract. This paper presents an interface model for interoperable educational courses among web, digital TV and mobile devices. Taken into consideration the current difficulties to standardize content among the three platforms and the requirements to create satisfactory interfaces for educational content, the main objective is to develop an interface model for learning objects which keeps at most the same visual and interactive characteristics, independently of where they are accessed. To validate the proposal, a course about health and life quality was implemented, with an interoperable interface aiming to fulfill these requirements.

Keywords: interface, education, interoperability

\section{Introdução}

Cada vez mais estamos vivendo em um mundo globalizado, onde a tecnologia ganha grande espaço na sociedade e permeia as mais diversas atividades, entre elas, a educação. Hoje em dia, não há como desvincular uma da outra, considerando-se que a tecnologia pode facilitar e enriquecer o processo de ensino-aprendizagem quando bem utilizada. Portanto, os materiais educacionais a serem desenvolvidos não poderiam mais ser pensados para uso exclusivo em um só ambiente. Há um grande número de recursos midiáticos e tecnológicos disponíveis para uso na educação, o que gera um desafio para toda uma área multidisciplinar que tem a função de construir e selecionar objetos de aprendizagem baseados em novos modelos educacionais.

Segundo Santos e Tarouco (2008), torna-se necessário revisar os paradigmas educacionais tradicionais para construir os novos, combinando-se, de maneira adequada, tecnologia com humanismo, e modernidade com cidadania. 
Castro (2009) descreve a proposta do governo brasileiro de criar o Centro Nacional de Excelência em Produção de Conteúdos Digitais Interativos e Interoperáveis para desenvolver projetos de produção de conteúdos audiovisuais digitais voltados a diferentes plataformas e também para convergência tecnológica, possibilitando seu uso em vários dispositivos tecnológicos simultaneamente. Nunca houve tanta necessidade de elaborar materiais que contemplem as tecnologias atuais e de nova geração, sempre levando em consideração o público alvo onde os produtos interativos poderão ser utilizados (PREECE, ROGERS \& SHARP, 2005).

Aproximadamente, o percentual de usuários que tem acesso à internet, televisão e celulares no Brasil é de, respectivamente, 18\%, 98\% e 81,60\% (TELECO, 2009) (NIC, 2009), e há uma natural convergência para o desenvolvimento de conteúdo que se adapte a esses três grandes mecanismos que dão acesso às tecnologias digitais, uma vez que construir conteúdo com o mesmo objetivo, porém de forma específica para cada um dos dispositivos, elevaria, desnecessariamente, o custo de criação e manutenção do mesmo (BORDIGNON et al., 2009).

O foco deste artigo é propor diretrizes para a concepção de interfaces de maneira que possam ser replicadas de forma adequada em cada um dos dispositivos no qual os objetos de aprendizagem são apresentados.

Considerando-se estes aspectos, as próximas seções estão divididas da seguinte maneira: a seção 2 apresenta as recomendações padronizadas de interoperabilidade, enquanto a seção 3 aborda questões referentes a uma interface adequada para um objetivo educacional. A seção 4 demonstra a implementação e os modelos de interface utilizados, a seção 5 apresenta os trabalhos relacionados e a seção 6 traz as considerações finais.

\section{Recomendações de Interoperabilidade}

Conceitua-se interoperabilidade como a capacidade de utilização de uma linguagem, padrão ou protocolo comum, independente do dispositivo, de forma que um mesmo conteúdo possa ser utilizado em diferentes ambientes (BORDIGNON et al., 2009). Porém, algumas recomendações são específicas para cada plataforma, conforme detalhado na Tabela 1 (W3C, 2008) (BBC, 2005) (ABNT, 2008) (3GPP, 2007).

Tabela 1 - Recomendações para cada plataforma

\begin{tabular}{|c|c|c|c|}
\hline Plataforma & Web & TV Digital & Celular \\
\hline Cor da Fonte & Cores escuras & Cores claras & Semelhante à web \\
\hline Cor de Fundo & Cores claras & Cores escuras & Semelhante à web \\
\hline $\begin{array}{c}\text { Fontes } \\
\text { Recomendadas }\end{array}$ & $\begin{array}{c}\text { Times New Roman, Geórgia } \\
\text { (com serifa); } \\
\text { Arial, Helvetica, Verdana } \\
\text { (sem serifa) }\end{array}$ & $\begin{array}{c}\text { Fontes não serifadas, como } \\
\text { Gill Sans, Tiresias, Arial e } \\
\text { Frutiger }\end{array}$ & $\begin{array}{c}\text { Cada navegador de } \\
\text { dispositivos móveis possui } \\
\text { sua definição de fonte. } \\
\text { Ex: Nokia é Sans-serif }\end{array}$ \\
\hline $\begin{array}{c}\text { Tamanho de } \\
\text { Fonte }\end{array}$ & $\begin{array}{c}\text { (Sem recomendações } \\
\text { específicas) } \\
\text { 12pt é apropriado para } \\
\text { leitura de tela }\end{array}$ & $\begin{array}{c}\text { Título: } 36 \mathrm{pt} \\
\text { Menus: } 20 \mathrm{pt} \\
\text { Texto: } 22 \mathrm{pt} \\
\text { Botões: } 18 \mathrm{pt}\end{array}$ & $\begin{array}{c}\text { Título 1: } 18 \text { px } \\
\text { Título 2: } 16 \text { px } \\
\text { Texto: } 12 \text { px }\end{array}$ \\
\hline Imagens & $\begin{array}{c}\text { (Sem recomendações } \\
\text { específicas) } \\
\text { Suporta vários formatos: } \\
\text { PNG, JPEG, GIF, etc. }\end{array}$ & $\begin{array}{c}\text { Formatos PNG, JPEG e } \\
\text { H.264/MPEG-4 AVC } \\
\text { "quadros-I" são mandatórios } \\
\text { para todos os receptores. }\end{array}$ & $\begin{array}{c}\text { Formatos GIF, JPEG e } \\
\text { PNG são suportados pela } \\
\text { maioria dos dispositivos. }\end{array}$ \\
\hline
\end{tabular}




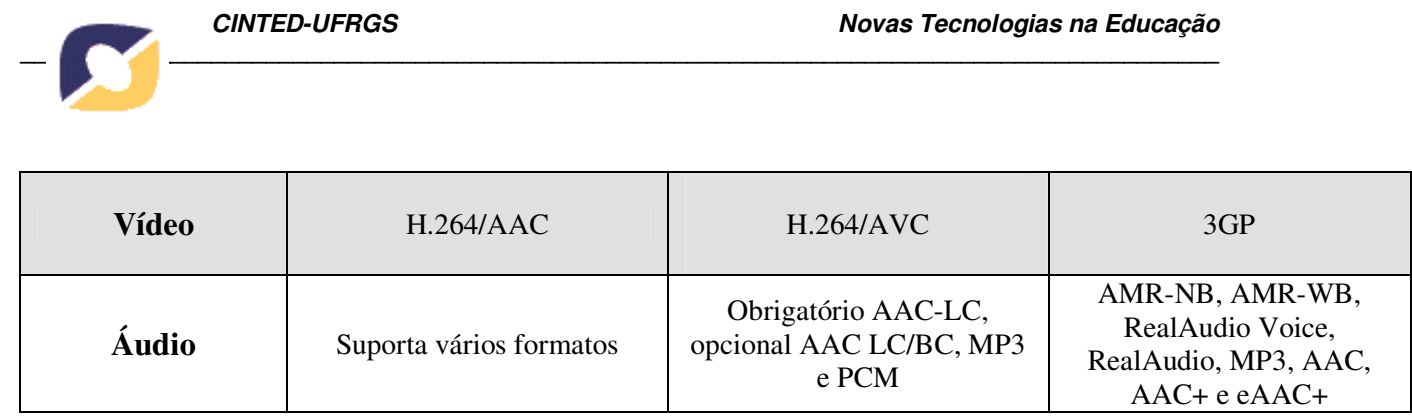

A partir da tabela é possível observar que, em praticamente todos os critérios, as recomendações são diferentes para cada uma das plataformas, o que efetivamente exige um trabalho de adaptação das interfaces de um dispositivo para outro.

\section{Interface Educacional}

Em um objeto de aprendizagem, é sua interface que possibilitará ao aluno ter acesso a todas suas funções, e sua qualidade pode afetar positivamente ou negativamente o desempenho do aprendiz. Portanto, é fundamental colocar a importância de se pensar no aluno e no objetivo do conteúdo instrucional ao se construir uma interface para um objeto educacional, pois é primordial que ele consiga usá-la sem problemas.

Chama-se de usabilidade a facilidade que os usuários têm em aprender a utilizar um produto para alcançar seus objetivos e o quão satisfeitos ficam com o processo. A avaliação dos critérios pode ser subjetiva, envolvendo, muitas vezes, diferentes aspectos e teorias. As próximas subseções apresentam alguns destes princípios.

\subsection{Teoria da Carga Cognitiva}

Vários estudos sobre o sistema cognitivo humano vêm sendo realizados nas últimas décadas. John Sweller, psicólogo australiano, com base na teoria do número mágico 7 (Miller, 1956), desenvolveu alguns princípios denominados de Teoria da Carga Cognitiva, aplicando-a a todos os tipos de conteúdos, mídias e estudantes, bem como às plataformas de aprendizagem a distância, objetivando potencializar a aprendizagem e desenvolver habilidades flexíveis através da criação e uso de recursos e ambientes de aprendizagem que estejam em sintonia com processo cognitivo humano (CLARK, R., NGUYEN, F., SWELLER, 2006).

Essa teoria define que um material instrucional armazena três tipos de cargas, denominadas intrínseca, relevante e irrelevante. A carga intrínseca diz respeito à facilidade ou dificuldade que temos em aprender determinado conteúdo, ou seja, está ligada à complexidade do material de ensino. A carga relevante trata da promoção de adquirir novos conhecimentos, uma vez que beneficia a aprendizagem com atividades de ensino apropriadas. Já a carga irrelevante é aquela que não se relaciona diretamente com o conteúdo a ser aprendido, e desperdiça recursos mentais que poderiam ser aproveitados de outra forma. Portanto, deve-se atentar para os seguintes critérios no desenvolvimento de um material instrucional: gerenciamento da carga cognitiva instrínseca; aumento da carga cognitiva relevante; e redução da carga cognitiva irrelevante.

No que diz respeito à interoperabilidade, é importante que na adaptação de um conteúdo de uma plataforma para outra, não se tenha um aumento de carga cognitiva irrelevante, ou uma redução de carga cognitiva relevante. 


\subsection{Leis de Gestalt}

As leis de Gestalt tratam sobre os padrões humanos de percepção, e procuram facilitar a compreensão de informações, uma vez que os elementos são agrupados conforme as características que possuem entre si. Gomes Filho (2000) apresenta as seguintes definições para as leis:

- Proximidade: elementos próximos no espaço são percebidos como pertencendo à mesma estrutura;

- Similaridade: elementos similares são igualmente compreendidos como fazendo parte de uma mesma estrutura;

- Simetria: elementos arrumados de forma simétrica são interpretados como uma unidade;

- Continuidade: a mente tende a dar continuidade a linhas além do ponto onde parece terminar;

- Simplicidade: elementos simples se destacam no fundo;

- Clausura (Fechamento): quando o desenho do elemento sugere uma extensão lógica, o cérebro tende a "fechar" o mesmo, completando visualmente a figura;

- Experiência: a percepção visual volta a experiências passadas, completando padrões visuais.

No que diz respeito à construção de interfaces interoperáveis, é importante observar que tais leis sejam mantidas em todas as plataformas. Por exemplo, elementos gráficos dispostos próximos uns dos outros em uma interface com menor espaço de visualização, dando a percepção de unidade, conservam tal percepção em uma interface com maior área de visualização.

\subsection{Utilização de imagens e animações}

Shimada e Kitajima (2006) apontam dois fatores que fazem com que ilustrações tenham um impacto positivo na aprendizagem, influenciando positivamente na construção do conhecimento: efeito de motivação, caracterizado pelo impulso que temos em realizar ou não uma ação (uma ilustração pode, por exemplo, nos motivar a ler um texto ou nos levar a ignorá-lo); efeito de elaboração, quando um indivíduo visualiza uma imagem junto a um texto, este cria uma representação da imagem e a associa ao conteúdo lido.

Portanto, as imagens são consideradas importantes recursos para a visualização e contribuem para a inteligibilidade de diversos materiais, sendo esses educacionais ou não. Além disso, desempenham um papel fundamental na constituição das idéias científicas e na sua conceitualização (MARTINS; GOUVEA; PICCINI, 2005). Portanto, na construção de interfaces interoperáveis, é importante que as imagens tenham visualização adequada em todas as plataformas na medida em que a própria imagem na maior parte do tempo faz parte do conteúdo educacional.

\subsection{Apresentação de textos}

Nielsen (1997) afirma que não se lê na web da mesma maneira que no meio impresso. Portanto, estendendo esse conceito, não se deve escrever para a web, TV Digital ou dispositivos móveis do mesmo jeito que para o meio impresso. Com relação às cores, cada uma possui uma sensação associada (cores frias e cores quentes) e seu uso indevido pode prejudicar a comunicação visual numa tela. Vermelho e roxo-violeta são cores quentes e luminosas que deveriam ser usadas apenas em alertas ou pequenos espaços. A cor de fonte preta sobre fundo amarelo âmbar é a combinação que dá maior 
contraste visual, sendo uma das poucas cores que tem a mesma visibilidade na inversão de fundo. No que diz respeito a contraste e cores complementares, explica-se que uma cor se intensifica quando justaposta ou à sua complementar, ou ao branco, preto e cinza, e reduz sua intensidade quando se mistura algo de sua complementar. O branco e preto puros permitem a realização de harmonia e contraste. $O$ excesso de exposição ao azul causa fadiga, apesar de ser uma cor que inspira paz e introspecção, já o verde é eficaz contra a irritabilidade, a fadiga e a insônia (BORGES et al.).

Em decorrência disso, a apresentação de textos nas telas dos dispositivos deve ser feita com alguns cuidados, conforme aspectos apresentados acima bem como padronizações descritas na seção 2 (Tabela 1).

\subsection{Layout de tela}

Nos dias de hoje, somos constantemente bombardeados por um conjunto de estímulos visuais. Nossa mente ignora a maior parte desses, processando apenas aqueles que recebem um significado especial baseado em nossa experiência anterior.

Krug (2005, p.22) aponta que não lemos as interfaces, mas rastreamos estas em busca de palavras ou frases que capturem nossa atenção. O tamanho, cor e disposição dos elementos na página influenciam a facilidade com que os localizamos. Elementos maiores são encontrados mais rapidamente, bem como elementos em cores saturadas que contrastam com o fundo da tela.

É também importante deixar uma quantidade razoável de espaços vazios, evitando a sobrecarga cognitiva. Experimentos mostram a maior parte dos usuários percebe a densidade de uma página da mesma maneira, sendo que o excesso de elementos reduz a performance na busca de informações (WARDEINER, 2007). Devido a isso, na produção de conteúdos para diferentes plataformas, é importante se trabalhar com interfaces mais limpas, minimizando a sobrecarga e possibilitando um foco maior no conteúdo do curso.

\subsection{Orientação}

Da mesma forma que podemos nos sentir perdidos quando circulamos em um local desconhecido, também podemos nos sentir desorientados ao utilizarmos uma interface, devido a uma sensação de insegurança e uma série de questões recorrentes: Onde estou? Que atividades já realizei? Quais os próximos passos? Como posso retornar?

Um bom sistema de orientação provê respostas a estas questões o tempo todo, sinalizando os caminhos percorridos pelo usuário e indicando com clareza outros disponíveis. Então, a produção de interfaces para diferentes plataformas tem o desafio de trazer uma percepção de orientação nítida para todos os dispositivos.

\subsection{Interação}

Um conteúdo educativo concebido com bases construtivistas, certamente tem na interação um dos seus principais focos. Com base nesta abordagem, os alunos constroem seu conhecimento a partir de suas próprias experiências e a partir de autoregulações que ocorrem através das relações estabelecidas entre o sujeito e o objeto (PIAGET, 1973). Neste sentido é fundamental conceber adequadamente os mecanismos de interação, promovendo o pensamento e a reflexão, e levando o usuário além da simples seleção de hiperlinks para avançar ou retornar na apresentação dos conteúdos (TOGNAZZINI, 2003). 
No que diz respeito à concepção de materiais educacionais multi-plataformas, um design de interação adequado é essencial, principalmente porque os dispositivos podem ser controlados por diferentes equipamentos, ou seja, a TV emprega o controle remoto como principal dispositivo de interação; o computador possui o teclado e o mouse como dispositivos de interação; e o celular dispõe de teclado, além de alguns modelos disporem de tela sensível ao toque. O problema que se impõe é que alguns destes dispositivos são mais adaptados do que outros para determinadas tarefas, e isso requer uma concepção cautelosa de qualquer tarefa interativa.

\subsection{Estética e Afetividade}

Emoção e motivação são forças centrais por trás de atos humanos. A pesquisa na área de motivação assume que esta está presente no desempenho de todas as atividades. Por esta razão, um bom material educativo explora não apenas o lado cognitivo, mas também o lado afetivo, e utiliza estratégias de motivação específicas.

Uma forma eficaz de se trabalhar afetividade é através de agentes de interface, ou seja, personagens virtuais capazes de perceber os estados afetivos do usuário e reagir a estes de maneira apropriada. Craig et al. (2002) mostraram que a interação com personagens estáticos e animados podem afetar a aprendizagem dos estudantes. Outros experimentos demonstraram que a presença da figura humana tem um efeito positivo nas experiências interativas dos estudantes, mostrando que estudantes consideraram um tópico em estudo menos difícil e a apresentação mais lúdica na presença de um personagem virtual (ANDRÉ e RIST, 2002). Neste sentido, é importante incorporar nas interfaces elementos que trabalhem estética e afetividade.

\section{Validação via interface do Curso Viva-Saudável}

O "Viva Saudável" é um objeto de aprendizagem sobre a melhoria da qualidade de vida através da alimentação saudável e da prática regular de atividade física. É um curso que visa promover a conscientização sobre a importância que esses dois hábitos têm na nossa saúde física e mental e foi pensado para jovens entre 12 e 15 anos, mas pode ser cursado por outras faixas etárias que tenham interesse no assunto, pois não possui censura. $\mathrm{O}$ objeto está dividido em três módulos, sendo que o estudo apresentado neste artigo aborda o módulo 1 , que tem por objetivo motivar os alunos à reflexão sobre seus hábitos alimentares e a importância do exercício físico. Prevê atividades como quiz, vídeo e testes para verificação de massa corporal.

$\mathrm{Na}$ Figura 1, pode-se observar a interface do índice do curso implementada em Web, TV Digital e dispositivos móveis, respectivamente.

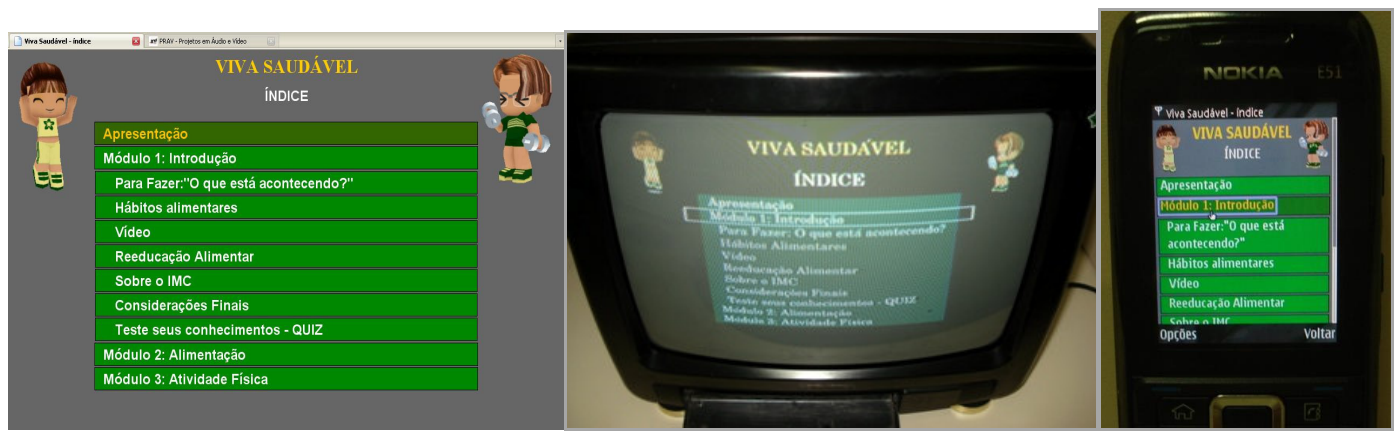

(a) Web (b) TV Digital (c) Celular

Figura 1 - Interface para índice 
É possível observar que as interfaces se assemelham bastante, mas apresentam tonalidades, fontes, layout e elementos interativos com algumas diferenças devido às limitações de cada plataforma. As subseções abaixo apresentam detalhes técnicos e em seguida discutem algumas das escolhas feitas de acordo com o referencial teórico apresentado anteriormente.

\subsection{Avaliação preliminar da interface}

A implementação das três propostas de interface foi realizada através de uma combinação de XHTML com folhas de estilo (CSS - Cascading Style Sheets). A opção pelo XHTML deu-se por ser este um padrão tanto para Web, como para TV Digital e telefones móveis, o que permite o acesso ao conteúdo através de navegadores sem maiores modificações. O CSS também pode ser utilizado para resolver os problemas de adaptação de cores e estilos via definição de um arquivo CSS para cada plataforma. Em relação às imagens, o padrão JPEG é aconselhado por ser compatível com as três plataformas. Para vídeos, optou-se pelo armazenamento em H.264 AVC para Web e TV Digital, e 3GP com compressão H.263 para dispositivos móveis, visto que não há um formato de vídeo comum para as plataformas estudadas. Para áudio, a alternativa adotada foi a geração e armazenamento de conteúdo em formato AAC, pois é padrão em Web, TV Digital e móveis.

A Figura 2 apresenta a segunda tela do módulo 1 (introdução), com a agente "Vivita" passando alguns conhecimentos e indicando a realização de uma atividade.

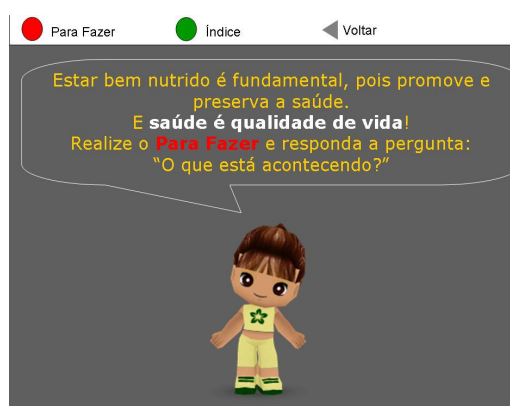

(a) Web

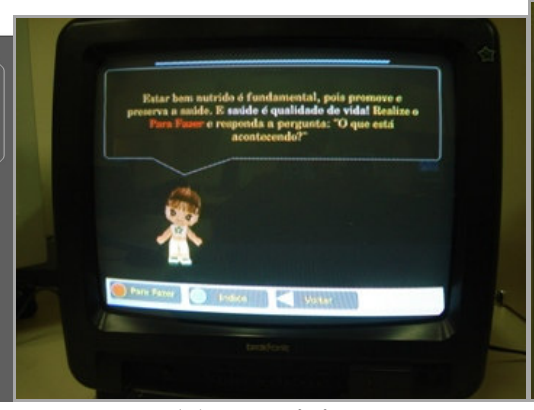

(b) TV Digital

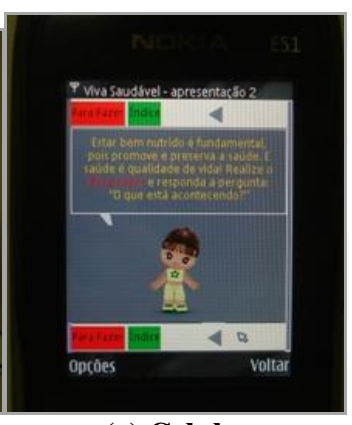

(c) Celular

Figura 2 - Interface da introdução do curso (tela 2)

Está representada, na Figura 3, a interface para a atividade "Na Família, O que está acontecendo?", onde o aluno tem a possibilidade de responder por quiz ou fórum, caso tenha acesso a interatividade global, ou seja, não deixará de fazer a atividade, pois responde ao quiz, mas pode ainda deixar sua opinião em um fórum de discussão de um ambiente colaborativo, tipo moodle.

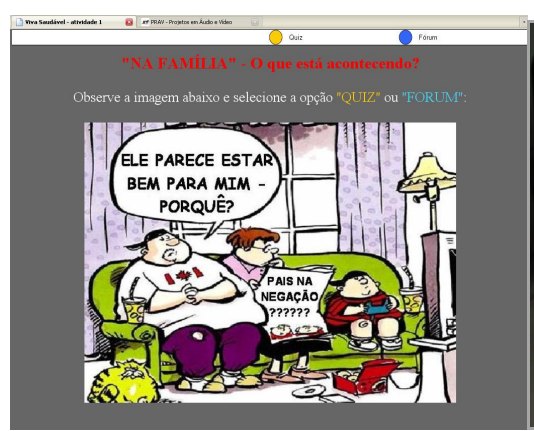

(a) Web

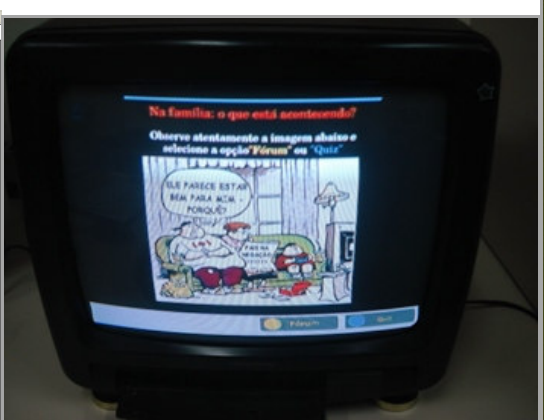

(b) TV Digital

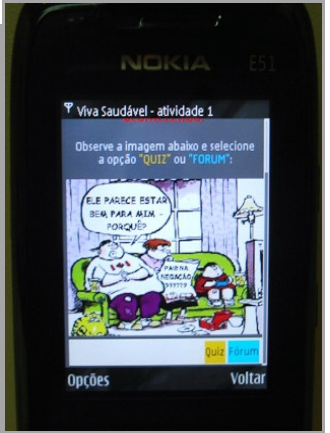

(c) Celular 
Figura 3 - Interface para a atividade "Para Fazer: Na Família, o que está acontecendo?"

Com base nas limitações das três plataformas, bem como nas teorias de confecção de interfaces, propõe-se a criação de um modelo que atenda o maior número de possibilidades.

Seguindo uma linha comum para o design gráfico do material educacional e adequando-se ao estudo de cores realizado por Borges et al. (2000), propõe-se aqui manter um padrão de tonalidades similar para as três plataformas, prevalecendo no fundo de tela a cor preta para TV Digital e tonalidades um pouco mais claras (cor cinza) para Web e celulares. Para cores de fonte estabeleceu-se o amarelo âmbar, com alternância de palavras em branco ou vermelho para destaque. A cor do índice do curso foi escolhida por questões estéticas e pelas sensações que tal cor transmite, pois verde é a cor da natureza, do frescor e da vida, além de representar a esperança. Para navegação, optou-se por setas de "voltar" e "avançar" e botões coloridos verde (índice), vermelho (atividade a ser realizada), além do azul (fórum) e amarelo (quiz) (Figuras 2 e 3).

As cores azul e amarela dos botões de navegação (Figura 3) estão relacionadas às cores do texto explicativo para "Fórum" (caso tenha interatividade) ou "Quiz". Como as cores verde e vermelha já foram utilizadas e representam, respectivamente, índice e algum tipo de atividade, optou-se pelas cores azul e amarelo por serem essas as tonalidades padronizadas no controle remoto da TV interativa (BBC, 2005), seguindo a mesma ordem de apresentação do mesmo. A opção de deixar cor e texto foi para acessibilidade a pessoas daltônicas e que, porventura, poderiam ter alguma dificuldade de reconhecer as cores dos botões. Como o aluno é orientado a fazer a atividade, não aparece a seta de "avançar" na Figura 2.

Com base nos aspectos relativos ao layout, poucos elementos foram colocados em cada tela, evitando problemas de sobrecarga de informação. A utilização de princípios da Gestalt também podem ser percebidos na construção da interface, como na figura 2 (Web e TV Digital), na qual vê-se o botão vermelho próximo ao seu rótulo (Para fazer), trazendo a percepção de unidade ao conjunto botão-rótulo.

Mecanismos de orientação e navegação eficazes permitem que os estudantes mantenham seu foco nos objetos de estudo e não precisem despender nenhum esforço para se localizar no software ou aprender a utilizá-lo. Pensando nisso, optou-se por manter aparentes somente as setas ou botões que o aluno pode usar, além de encontrálos sempre no mesmo lugar e com as mesmas cores. Porém, um diferencial quanto ao posicionamento da barra de navegação é encontrado em motivo das recomendações para cada plataforma (BBC, 2005) (W3C, 2008). Nas Figuras 2 e 3, pode-se observar que para web é superior, para TV Digital é inferior e para celular, em virtude da utilização de rolagem para visualização a todo conteúdo, a barra aparece em cima e embaixo, permitindo ao usuário interagir sem a necessidade de "navegar" para cima ou para baixo. Com relação ao aspecto afetividade, este é desempenhado pelos personagens Vivita e Betinho, que apresentam dicas ao usuário e buscam, através de sua presença, estabelecer vínculos sociais, ainda conferindo ao objeto um caráter lúdico.

\section{Trabalhos Relacionados}

Oliveira e Santos (2005) apresentam um estudo de usabilidade da interface gráfica de um programa de geometria dinâmica chamado iGEOM, procurando avaliar a atual interface e propor uma nova, mais adequada para o ensino da geometria descritiva. Constataram alguns problemas quanto à remoção de elementos ou ícones sem notificação prévia e que poderiam ser corrigidos sem qualquer esforço, além da falta de 
controle da área de trabalho, colocando o usuário na obrigação de um treinamento prévio, ou seja, algo muito comum, mas que deveria ser impensável na hora de elaborar material multimídia dentro de uma reflexão pedagógica. Porém, o estudo não apresenta opção da nova interface ser interoperável.

Tizzei et al. (2008) apresentam o projeto de um repositório de componentes de software reutilizáveis, sendo que o mesmo pretende ser interoperável com outros ambientes de desenvolvimento através de uma interface previamente especificada, mas a interoperabilidade desse estudo limita-se a ambientes que utilizem interface web, onde optou-se adotar o padrão RAS para facilitar a troca de dados.

\section{Considerações Finais}

A produção de conteúdos digitais interativos e interoperáveis é uma necessidade crescente, tornando-se de extrema importância a utilização de diretrizes específicas para cada plataforma e para o design das interfaces, direcionadas à promoção da aprendizagem.

A principal contribuição deste artigo foi o desenvolvimento e a implementação de um modelo de interface para objetos de aprendizagem que visa manter as mesmas características visuais e interativas independente da plataforma que forem acessados, no caso, Web, TV Digital e dispositivos móveis.

Este estudo reforça a necessidade de conscientização, por parte dos produtores de conteúdo, da importância de criar uma interface única e multiplataforma, visando atingir o maior número de usuários. Com tal interface implementada, outros benefícios surgem, como a facilidade de manutenção (visto que o conteúdo é único), e a otimização de armazenamento (visto que o mesmo conteúdo é reutilizado para todas as plataformas).

\section{Referências}

ABNT. ABNT NBR 15604. Televisão Digital Terrestre - Receptores. 2008. Disponível em http://www.abnt.org.br/tvdigital/. Acesso em maio de 2009.

ANDRÉ, E. \& RIST, T. From adaptive hypertext to personalized web companions. Communications of the ACM, v45 n5, May. 2002.

BBC. Designing for interactive television $\mathrm{v}$ 1.0. BBCi \& Interactive tv programmes. 2005.

BORDIGNON, Alexandro; SIMIONI, Adriano; VARELLA, Fernando; TOSS, Júlio; BARBOSA, Maria. L. K.; ROESLER, Valter. Mechanisms for Interoperable Content Production among Web, Digital TV and Mobiles. $9^{\text {th }}$ World Conference on Computers in Education. Bento Gonçalves, RS. WCCE 2009.

BORGES, Roberto C. M.; WINKLER, Marco A.; BASSO, Karen. Considerações sobre Cores na Web. IHC 2000, Gramado, 2000.

CASTRO, Cosette. Uso de plataformas tecnológicas para inclusão digital - o caso da TV Digital e da produção de conteúdo. Revista Inclusão Social, Brasília, v. 3, n. 1, p. 70-74, out. 2007/mar. 2008.

CLARK, R., NGUYEN, F., SWELLER, J. Efficiency in Learning: Evidence-based Guidelines to Manage Cognitive Load. San Francisco: Pfeiffer, 2006. 
CRAIG, S., GHOLSON, B. e DRISCOLL, D. Animated Pedagogical Agents in Multimedia Educational Environments. Journal of Educational Psychology, Vol. 94, No. 2, 428-434. 2002.

GOMES FILHO, João. Gestalt do Objeto - Sistema de Leitura Visual da Forma. São Paulo, SP. Escritura Editoras, 2000.

KRUG, Steve. Don't Make Me Think: A Common Sense Approach to Web Usability ( $2^{\text {nd }}$ Edition). New Riders Press, 2005.

MARTINS, Isabel; GOUVÊA, Guaracira; PICCINI, Cláudia. Aprendendo com Imagens. Disponível em http://cienciaecultura.bvs.br/scielo.php?pid=S000967252005000400021\&script=sci_arttext\&tlng=en. Acesso em maio de 2009.

MILLER, George A. (1956). The Magical Number Seven, Plus or Minus Two: Some Limits on Our Capacity for Processing Information. Disponível em http://www.musanim.com/miller1956/. Acesso em maio de 2009.

NIC. Network Information Center. TIC Domicílios 2008. Disponível em http://www.nic.br/imprensa/releases/2009/rl-2009-05.pdf. Acesso em maio de 2009.

NIELSEN. Jakob. (1997). How users read on web. Alertbox, October, 1. Disponível em http://www.useit.com/alterbox/9710a.html. Acesso em maio de 2009.

OLIVEIRA, André L. L.; SANTOS, Eduardo T. Estudo de Usabilidade da Interface Gráfica do Programa de Geometria Dinâmica $i$ GEOM. V International Conference on Graphics Engineering for Arts nd Design. Santa Cruz do Sul, RS. 2003

PREECE, Jennifer; ROGERS, Yvonne; SHARP, Helen. Design de Interação. Além da interação homem-computador. Porto Alegre, RS. Bookman, 2005.

PIAGET, Jean. Estudos Sociológicos. Rio de Janeiro: Forense. 243p. 1973.

SANTOS, Leila M. A.; TAROUCO, Liane M. R. A Contribuição dos Princípios da Teoria da Carga Cognitiva para uma Educação mediada pela Tecnologia. In V Congresso Brasileiro de Ensino Superior a Distância. Gramado, RS. ESUD, 2008.

SHIMADA, H. e KITAJIMA, M. Why Do Illustrations Promote Text Comprehension? Motivation Effect and Elaboration Effect. 5th International Conference of the Cognitive Science. Vancouver, British Columbia, Canadá, July 26, 2006.

TELECO. Informações em Telecomunicações. Disponível em http://www.teleco.com.br/ncel.asp. Acesso em maio de 2009.

TIZZEI, Leonardo P.; PINTO, Helder S.; GUERRA, Paulo A. C.; RUBIRA, Cecília M. F. Um Repositório de Componentes com Suporte à Evolucão centrada na Arquitetura de Software. Workshop de Desenvolvimento Baseado em Compnentes. Juiz de Fora, MG. WDBC 2005.

TOGNAZZINI, B. (2003). First Principles of Interaction Design. AskTog / Nielsen and Norman Group. Disponível em http://www.asktog.com/basics/firstPrinciples.htm.

Acesso em junho de 2009.

WARDEINER, M. R. (2007). Usability Engineering Team: Design Guidelines.

NASA Glen Research Center. Disponível em:

http://www.grc.nasa.gov/WWW/usability/navigatecss.html. Acesso em maio de 2009.

W3C. Mobile Web Best Practices 1.0, Basic Guidelines. W3C Recommendation. 2008. Disponível em http://www.w3.org/TR/mobile-bp/. Acesso em maio de 2009. 
r

3GPP. The 3rd Generation Partnership Project. TS 26.244. 3GPP file format (3GP). 2007. Disponível em http://www.3gpp.org/ftp/Specs/html-info/26-series.htm. Acesso em maio de 2009. 